\title{
RESEARCH
}

\section{Androgen modulation of XBP1 is functionally driving part of the AR transcriptional program}

\author{
Suzan Stelloo1,*, Simon Linder1,*, Ekaterina Nevedomskaya1,2, Eider Valle-Encinas', Iris de Rink³, \\ Lodewyk F A Wessels ${ }^{2,4}$, Henk van der Poel5, Andries M Bergman ${ }^{6,7}$ and Wilbert Zwart ${ }^{1,8}$ \\ 'Division of Oncogenomics, Oncode Institute, The Netherlands Cancer Institute, Amsterdam, The Netherlands \\ 2Division of Molecular Carcinogenesis, Oncode Institute, The Netherlands Cancer Institute, Amsterdam, The Netherlands \\ ${ }^{3}$ Genomics Core Facility, The Netherlands Cancer Institute, Amsterdam, The Netherlands \\ ${ }^{4}$ Faculty of EEMCS, Delft University of Technology, Delft, The Netherlands \\ ${ }^{5}$ Division of Urology, The Netherlands Cancer Institute, Antoni van Leeuwenhoek Hospital, Amsterdam, The Netherlands \\ ${ }^{6}$ Division of Oncogenomics, The Netherlands Cancer Institute, Amsterdam, The Netherlands \\ 7Division of Medical Oncology, The Netherlands Cancer Institute, Amsterdam, The Netherlands \\ ${ }^{8}$ Laboratory of Chemical Biology and Institute for Complex Molecular Systems, Department of Biomedical Engineering, Eindhoven University of \\ Technology, Eindhoven, The Netherlands
}

Correspondence should be addressed to A M Bergman or W Zwart: a.bergman@nki.nl or w.zwart@nki.nl

*(S Stelloo and S Linder contributed equally to this work)

\begin{abstract}
Prostate cancer development and progression is largely dependent on androgen receptor (AR) signaling. AR is a hormone-dependent transcription factor, which binds to thousands of sites throughout the human genome to regulate expression of directly responsive genes, including pro-survival genes that enable tumor cells to cope with increased cellular stress. ERN1 and XBP1 - two key players of the unfolded protein response (UPR) - are among such stress-associated genes. Here, we show that XBP1 levels in primary prostate cancer are associated with biochemical recurrence in five independent cohorts. Patients who received AR-targeted therapies had significantly lower XBP1 expression, whereas expression of the active form of XBP1 (XBP1s) was elevated. In vitro results show that AR-induced ERN1 expression led to increased XBP1s mRNA and protein levels. Furthermore, ChIP-seq analysis revealed that XBP1s binds enhancers upon stress stimuli regulating genes involved in UPR processes, elF2 signaling and protein ubiquitination. We further demonstrate genomic overlap of AR- and XBP1s-binding sites, suggesting genomic conversion of the two signaling cascades. Transcriptomic effects of XBP1 were further studied by knockdown experiments, which lead to decreased expression of androgen-responsive genes and UPR genes. These results suggest a two-step mechanism of gene regulation, which involves androgen-induced expression of ERN1, thereby enhancing XBP1 splicing and transcriptional activity. This signaling cascade may prepare the cells for the increased protein folding, mRNA decay and translation that accompanies AR-regulated tumor cell proliferation.
\end{abstract}

\section{Key Words}

- androgen receptor

- XBP1 splicing

- ChIP-seq

- unfolded protein response https://erc bioscientifica.com https://doi.org/10.1530/ERC-19-0181
2020 Society for Endocrinology Published by Bioscientifica Ltd. Printed in Great Britain
Endocrine-Related Cancer (2020) 27, 67-79 


\section{Introduction}

In eukaryotic cells, homeostasis between protein production and degradation is a tightly regulated process. This regulation is perturbed when unfolded or misfolded proteins build up in the endoplasmic reticulum (ER), which induces the unfolded protein response (UPR). Upon UPR activation, a distinct transcriptional program is induced that increases the production of chaperones and foldases to restore homeostasis. The UPR plays a key role in a large spectrum of diseases, including neurodegenerative disorders, and this signaling cascade is also critically involved in many tumor types, including prostate cancer.

Prostate cancer development and progression is largely dictated by androgen receptor (AR) action: a hormone-dependent transcription factor that binds the genome at thousands of sites, regulating gene programs involved in tumor cell proliferation. There is growing evidence of a link between the UPR and AR function, in which AR directly regulates the expression of ER stressassociated genes, including endoplasmic reticulum to nucleus signaling 1 (ERN1) and X-box binding protein (XBP1) (Sheng et al. 2015, Erzurumlu \& Ballar 2017).

XBP1 is a basic leucine zipper (bZIP) transcription factor, triggered by the onset of ER stress and other physiological processes such as lipid and cholesterol metabolism, energy control, inflammation and cell differentiation (Hetz et al. 2013). Beyond AR signaling in prostate cancer, $X B P 1$ gene expression is upregulated by other factors of the endocrine system, including parathyroid hormone stimulation in osteoblasts (Zambelli et al. 2005), growth hormone in adipocytes (Huo et al. 2006) and estradiol stimulation in breast cancer cells (Sengupta et al. 2010). In addition to transcriptional control of $X B P 1$, its function is also posttranscriptionally regulated through inositol-requiring enzyme 1a (IRE1a), which is encoded by the ERN1 gene (hereafter both the gene and protein are referred to as ERN1, for simplicity). When ERN1 senses stress, its RNase function is activated, allowing excision of 26 nucleotides of the XBP1 mRNA which leads to a frameshift (Yoshida et al. 2001, Calfon et al. 2002, Hetz et al. 2013, Duarte et al. 2019). Consequently, XBP1 exists in two isoforms: XBP1 unspliced (XBP1u) and XBP1 spliced (XBP1s). Both isoforms contain a DNA-binding domain, but only XBP1s has a transactivation domain enabling transcriptional induction of UPR genes including DNAJB9 and SEC11C (Yoshida et al. 2001, Lee et al. 2003, Hassler et al. 2015). Even though the genomewide chromatin-binding features of XBP1 remain elusive in the context of prostate cancer, ChIP-on-ChIP and ChIP-seq analyses of XBP1 have been performed in other systems including skeletal muscle cells, breast cancer cells and liver tissue (Acosta-Alvear et al. 2007, Chen et al. 2014, Argemi et al. 2017). In these cells, XBP1 mainly binds promoters that contain ER stress response elements (ERSE: CCACG) or unfolded protein response elements (UPRE: ACGT) (Acosta-Alvear et al. 2007, Chen et al. 2014, Argemi et al. 2017). Besides regulating genes to maintain ER function, XBP1 also drives cell-type-specific gene expression profiles, such as the MIST gene in the myoblast cell line C2C12 to drive myogenic differentiation (Acosta-Alvear et al. 2007). Whether XBP1 induces a prostate-specific transcriptional program remains unknown.

In prostate cancer, $X B P 1$ expression levels vary at different stages of the disease. XBP1 expression is higher in primary prostate cancer compared to benign tissue, while its expression is lower in metastases relative to primary lesions (Takahashi et al. 2002, CuperlovicCulf et al. 2010, Stelloo et al. 2015). Furthermore, XBP1 knockdown reduced the growth of prostate cancer cell lines, irrespective of AR expression (Thorpe \& Schwarze 2010, Sheng et al. 2015, 2019).

We previously identified a prognostic 9-gene classifier for prostate cancer outcome, which included XBP1 (Stelloo et al. 2015). In this study, we further evaluated the prognostic value and biological function of XBP1 in prostate cancer. Low expression of XBP1 was consistently associated with biochemical progression in five independent cohorts. Using mRNA samples from a cohort of prostate cancer patients receiving neoadjuvant enzalutamide treatment, we could show that AR-targeted therapy reduced total XBP1 expression, while XBP1s levels were increased. Furthermore, in vitro assays showed that expression of both ERN1 and XBP1 and consequently XBP1 splicing is increased upon AR activation. ChIP-seq analysis revealed that spliced XBP1 binds the chromatin largely at promoters in the absence of hormonal cues, while AR activation induces XBP1s binding at enhancers that are co-occupied by AR to regulate genes involved in UPR processes and AR action. These findings illustrate a two-step mechanism of gene regulation, in which AR signaling through XBP1s primes the proliferating prostate tumor cell for increased protein folding, mRNA decay and protein translation.

\section{Materials and methods}

\section{Survival analysis}

Gene expression as well as clinical data of five cohorts (Glinsky et al. 2004, Taylor et al. 2010, Boormans et al. (c) 2020 Society for Endocrinology Published by Bioscientifica Ltd. Printed in Great Britain 
2013, Gulzar et al. 2013, Cancer Genome Atlas Research Network 2015) were used. Expression data from Boormans et al., Gulzar et al. and Taylor et al. were downloaded from the Gene Expression Omnibus (GEO) database (https://www.ncbi.nlm.nih.gov/geo/). Clinical data from Boormans et al. was received directly from the authors, while the Gulzar and Taylor datasets were obtained from the respective publication (Taylor et al. 2010, Gulzar et al. 2013). Expression and clinical data from Glinsky et al. were received directly from the authors, while the TCGA data were downloaded using the UCSC Xena browser (https:// xenabrowser.net). Samples were categorized into either high or low XBP1 expression using the median expression. Prognostic value of XBP1 expression was assessed using hazard ratios with CIs of 95\%, following the Cox's proportional hazard model (R package survival; https:// cran.r-project.org/package=survival). Meta-analysis was performed using the $\mathrm{R}$ package rmeta (https://cran.rproject.org/package=rmeta). Hazard ratios were combined using the fixed-effect model. Statistical relation between XBP1 expression and clinicopathological parameters, including Gleason Score, T-Stage, presence of lymph node metastasis and PSA level was tested using Pearson's $\chi^{2}$ test. For XBP1 splicing quantification, BAM files containing the XBP1 region were downloaded from the Genomics Data Commons (GDC) portal (https://portal.gdc.cancer.gov). Reads were converted to fastq format and aligned with hisat2 (version 2.0.4) using a bed file containing all splice variants of XBP1. The number of reads uniquely mapped to the complete gene region as well as only counts related to exon 4 were determined using Icount. Reads that exactly jumped from base position 1569 to 1595 within the XBP1 gene on chromosome 22 were determined using samtools. The number of reads were divided by the total number of reads per sample, and XBP1s-specific reads were normalized to total XBP1 reads, providing relative XBP1s expression values.

\section{DARANA patient samples}

Pre- and post-enzalutamide-treated prostate cancer tissue of trial cases were acquired from the DARANA study (Dynamics of androgen receptor genomics and transcriptomics after neoadjuvant androgen ablation, https://clinicaltrials.gov/ct2/show/NCT03297385). Patient characteristics prior to treatment are summarized in Supplementary Table 5 (see section on supplementary materials given at the end of this article). All trial patients received 3 months of neoadjuvant enzalutamide prior to radical prostatectomy. The study was approved by the
IRB of the Netherlands Cancer Institute, and informed consent was signed by all participants who entered the study, and all research was performed in accordance with relevant guidelines and regulations. Biopsy (pre-treatment samples) and prostatectomy specimens (post-treatment samples) were formalin fixed and paraffin embedded (FFPE). FFPE material was sectioned prior to RNA isolation and sections were pathologically assessed for tumor cell percentage. Only samples with a tumor cell percentage of $\geq 50 \%$ were used for further downstream analyses.

\section{Cell culture}

VCaP and LAPC4 cells were a kind gift from Dr Wytske M van Weerden (Erasmus MC) and Dr Michiel van der Heijden (NKI), respectively. Human prostate cancer cell lines were cultured in RPMI-1640 medium (LNCaP, VCaP) or IMDM medium (LAPC4) supplemented with 10\% FBS. All cell lines were authenticated by STR profiling (BaseClear, Leiden, The Netherlands). In addition, cell lines were stocked at early passages and used at $<20-25$ subcultures. For hormone deprivation, cells were cultured in medium containing $10 \%$ charcoal-treated FBS for 3 days, and subsequently treated with 10 nM R1881 (synthetic androgen, PerkinElmer) or vehicle (DMSO) and harvested at the indicated time points. For Thapsigargin (Tg, Sigma) stimulations, cells were treated with $10 \mathrm{nM} \mathrm{Tg}$ for $3 \mathrm{~h}$. For ERN1 inhibitor (MKC8866, Mechem Express) treatment, cells were hormone deprived as described previously and subsequently treated with 10 nM R1881 supplemented with either vehicle (DMSO) or 10 $\mu \mathrm{M}$ ERN1 inhibitor for $24 \mathrm{~h}$.

\section{Generation of ERN1-knockout cells with CRISPR-Cas9}

Guide RNA targeting human ERN1 (TCGGGTTTTGGTGTCGTACA) was cloned into the PX330 CRISPR Cas9 plasmid (Harmsen et al. 2018). LNCaP cells were transiently transfected using Lipofectamine 2000 transfection reagent (Invitrogen) according to the manufacturer's instructions. After transfection, cells were selected using puromycin $(0.5 \mu \mathrm{g} / \mathrm{mL})$, and single clones were picked and analyzed by TIDE (Tracking of Indels by Decomposition) (Brinkman et al. 2014) and Western blot. Finally, the clone with the best editing efficiency was selected.

\section{Transient transfections}

Lipofectamine 2000 was used for all transient transfections in LNCaP and LAPC4 cells. siRNA oligos targeting the androgen receptor (MU-003400-02), XBP1 (MU-00955202), and the non-targeting control (D-001206-14) were 
purchased from Dharmacon. GFP and XBP1s expression plasmids were kindly provided by Dr Sjaak Neefjes (LUMC) and Dr Laurie H Glimcher (Dana-Farber Cancer Institute), respectively. XBP1u containing expression plasmid was obtained from the CCSB-Broad Lentiviral Expression Library (https://horizondiscovery.com/en/ products/gene-modulation/overexpression-reagents/orfs/ pifs/CCSB-Broad-Lentiviral-Expression-Collection). In hormone deprivation experiments, $24 \mathrm{~h}$ after switching to charcoal-treated FBS, cells were transfected and further hormone deprived for additional 48-72 h.

\section{RNA isolation and mRNA expression analysis}

Total RNA from cell lines was isolated with TRIzol according to the manufacturer's protocol (Invitrogen). RNA from FFPE material was isolated from 2 to 10 sections of $10 \mu \mathrm{m}$ using the AllPrep DNA/RNA FFPE kit (Qiagen). cDNA was synthesized from $\sim 2 \mu \mathrm{g}$ (cell line) or 250 ng (FFPE samples) RNA using SuperScript III Reverse Transcriptase (Invitrogen) with random hexamer primers. qPCR was performed with SensiMix ${ }^{\mathrm{TM}}$ SYBR $^{\circledR}$ No-ROX Kit (GC Biotech). Relative mRNA expression levels were normalized to TBP (cell lines) or to the average gene expression of the three housekeeping genes $\beta$-actin, TBP and GAPDH (FFPE samples). Primer sequences are listed in Supplementary Table 1.

For RNA-seq, strand-specific libraries were generated with the TruSeq stranded mRNA sample preparation kit (Illumina, Part \# 15031047 Rev. E) and sequenced on a HiSeq2500. Sequencing data were aligned to hg38 using TopHat, and the number of reads per gene were measured with HTSeq count. Standard output of the Tophat was used to get the number of reads that span the 26nt splice junction in exon 4 of XBP1. EdgeR-Limma workflow was used for gene expression analysis (Robinson et al. 2010, Ritchie et al. 2015). Genes with $>1$ count per million in at least two samples were included. Gene set enrichment analysis (GSEA) was performed with the GSEA software (http://www.broad.mit.edu/gsea/index.jsp) using the MSigDB Hallmarks gene set collection. Ingenuity Pathway Analysis (IPA) software was used to identify upstream regulators of the differentially expressed genes (cutoff: absolute $\log _{2}$ fold change $>0.5$ ) .

\section{Chromatin immunoprecipitation}

Chromatin immunoprecipitations (ChIP) were performed as previously described (Stelloo et al. 2018). Nuclear lysates were incubated with $7.5 \mu \mathrm{g}$ of XBP1s antibody (Biolegend, 619502) prebound to $75 \mu \mathrm{L}$ protein A beads (Thermo Fisher Scientific, 10008D). Immunoprecipitated DNA was processed for library preparation (Part\# 0801-0303, KAPA biosystems kit). Samples were sequenced using an Illumina Hiseq2500 genome analyzer (65 bp reads, singleend) and aligned to the Human Reference Genome (hg19, February 2009). Reads were filtered based on MAPQ quality (quality $\geq 20$ ) and duplicate reads were removed. Peak calling over input control (input samples from publicly available datasets were merged and downsampled (Stelloo et al. 2018)) was performed using Dfilter and MACS peak callers (Zhang et al. 2008, Kumar et al. 2013). MACS was run with the default parameters and $P$ value $=10^{-5}$. DFilter was run with $b s=50, k s=30$, refine, nonzero. The peaks shared by both peak callers and both replicates were used for downstream analysis. Read counts and the number of aligned reads are shown in Supplementary Table 2 . Genome browser snapshots, heatmaps and density plots were generated using EaSeq (Lerdrup et al. 2016). Genomic region enrichment analysis and motif analysis were performed with CEAS (Shin et al. 2009) and the SeqPos motif tool (http://cistrome.org/ap/), respectively. The Diffbind R package was used to generate the correlation heatmap and PCA plot based on occupancy (Ross-Innes et al. 2012). All ChIP-seq data generated in this study are deposited in the GEO database under the accession GSE121880. Publicly available AR (GSE94682), H3K4me3 (GSM503907) and H3K27ac (GSM686937) ChIP-seq data from LNCaP cells were used.

For ChIP-seq validations, qPCR analyses were performed using SensiMix ${ }^{\mathrm{TM}}$ SYBR $^{\circledR}$ No-ROX Kit (GC Biotech). ChIP-qPCR enrichment was normalized to a negative control region. Primer sequences are listed in Supplementary Table 1.

ChIP-seq data are available through GEO accession number GSE121880 (https://www.ncbi.nlm.nih.gov/geo/ query/acc.cgi?acc=GSE121880).

\section{Western blotting}

Cells were lysed in Laemmli buffer supplemented with complete protease inhibitor cocktail. After protein separation via SDS-PAGE and protein transfer, membranes were incubated with antibodies against AR (sc-816, Santa Cruz Biotechnology), Actin (MAB1501R, Millipore), ERN1 (14C10 \#3294, Cell Signaling Technology), total XBP1 (GTX113295, GeneTex) or XBP1s (619502, Biolegend).
(C) 2020 Society for Endocrinology Published by Bioscientifica Ltd. Printed in Great Britain 


\section{Statistical analyses}

For statistical analyses of survival data, Cox regression models were used. To test statistical association of XBP1 expression with clinicopathological parameters, Pearson's $\chi^{2}$ test was used. XBP1(s) expression in pre- vs posttreatment samples were compared using paired t-tests. For qPCR data, unpaired t-tests were used to compare normalized gene expression levels upon XBP1u/XBP1s overexpression to control-transfected samples (GFP) and to compare R1881-stimulated or siXBP1-transfected cells to vehicle or siC control samples, respectively. Two-way ANOVAs followed by Tukey's multiple comparisons tests were used to compare siC to siAR upon vehicle (DMSO) or R1881 stimulation. For RNA-seq, XBP1 knockdown efficiency upon siRNA treatment was tested using a paired $t$-test. All error bars represent s.D.

\section{Results}

\section{XBP1 expression is correlated with disease progression and response to hormone therapy}

We previously identified a prognostic 9-gene signature, which among other genes included the transcription factor XBP1 (Stelloo et al. 2015). To further study the biological role of XBP1 in prostate cancer, we first evaluated the expression of XBP1 for prognostic relevance as a single gene by performing a meta-analysis of five publicly available datasets (743 cases and 170 events, in total). The patients were divided into low and high XBP1 expression groups on the basis of the median XBP1 expression within each cohort. We then tested the association of XBP1 expression with biochemical progression after radical prostatectomy. The combined hazard ratio (HR) for the five cohorts in univariate analysis is 0.52 (95\% CI: 0.38-0.72), indicating that low expression of XBP1 is associated with higher chance of biochemical recurrence in patients diagnosed with primary prostate cancer (Fig. 1A and Table 1). The relationship between
XBP1 expression and clinicopathological parameters for each cohort is summarized in Supplementary Table 3. Low XBP1 expression is associated with higher Gleason score, tumor stage and presence of lymph node metastasis in the TCGA cohort, while none of the clinical parameters reached significance in the other smaller cohorts.

As microarray probes do not distinguish between XBP1u and XBP1s, we further explored the RNAsequencing data from the TCGA cohort. XBP1s expression was quantified by measuring the number of reads spanning the $26 \mathrm{bp}$-long spliced region. Patients with low-XBP1s-expressing tumors have similar biochemical progression-free survival compared to patients with high-XBP1s-expressing tumors (logrank $P$ value 0.476 , Supplementary Fig. 1A). Also, none of the clinical parameters were associated with XBP1s transcript levels (Supplementary Table 4).

As the 9-gene signature is based on differential AR chromatin binding between primary prostate tumors and tumors with an acquired resistance to hormone therapy, we examined the expression of the nine genes in tumors from seven patients with locally advanced/metastatic prostate cancer before androgen deprivation therapy (ADT) and 22 weeks after therapy initiation (Rajan et al. 2014). The clustering of the pre- and post-therapy samples by gene expression is displayed in a PCA biplot, showing that DNER and XBP1 expression are the two major contributors to the variance between pre- and post-therapy samples (Fig. 1B). Expression of XBP1 is downregulated following ADT (Supplementary Fig. 1B), which is in concordance with a recently reported neoadjuvant ADT study (Sowalsky et al. 2018). To study XBP1s in a neoadjuvant setting, we analyzed the expression of XBP1 by qPCR in 29 paired pre-treatment core needle biopsy samples and post-treatment surgical resection samples from patients who received neoadjuvant enzalutamide for 3 months (DARANA - NCT03297385). In this series, we confirmed the marked downregulation of XBP1 in posttreatment samples compared to matched pre-treatment biopsies (Fig. 1C). Interestingly, XBP1s mRNA levels were

Table 1 Results of Cox regression model.

\begin{tabular}{|c|c|c|c|c|c|c|c|c|c|c|c|}
\hline Dataset & Accession no. & $\begin{array}{c}\text { No. } \\
\text { patients }\end{array}$ & $\begin{array}{c}\text { No. } \\
\text { events }\end{array}$ & Coef & $\operatorname{Exp}$ (coef) & S.E. (coef) & $\mathbf{z}$ & $\operatorname{Pr}(>|z|)$ & $\begin{array}{c}\text { Lower } \\
0.95\end{array}$ & $\begin{array}{c}\text { Upper } \\
0.95\end{array}$ & P logrank \\
\hline Taylor & GSE21034 & 131 & 27 & -0.79 & 0.45 & 0.40 & -1.97 & 0.05 & 0.21 & 1.00 & 0.05 \\
\hline TCGAa & - & 424 & 83 & -0.53 & 0.59 & 0.23 & -2.34 & 0.02 & 0.38 & 0.92 & 0.02 \\
\hline Gulzar & GSE40272 & 82 & 19 & -1.46 & 0.23 & 0.56 & -2.59 & 0.01 & 0.08 & 0.70 & 0.00 \\
\hline Glinskyb & - & 80 & 38 & -0.59 & 0.55 & 0.33 & -1.79 & 0.07 & 0.29 & 1.06 & 0.07 \\
\hline Boormans & GSE41408 & 48 & 28 & -0.34 & 0.71 & 0.38 & -0.89 & 0.38 & 0.34 & 1.51 & 0.37 \\
\hline
\end{tabular}

aCancer Genome Atlas Research Network (2015). bGlinsky et al. (2004). 


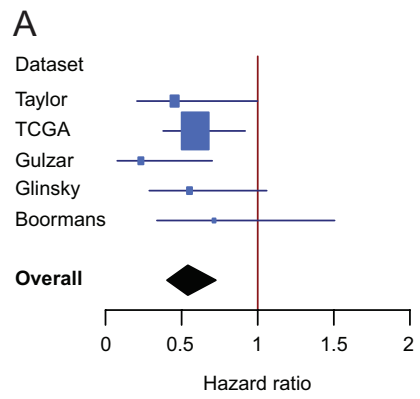

Unadjusted meta $\mathrm{HR}=0.54(0.40-0.72$
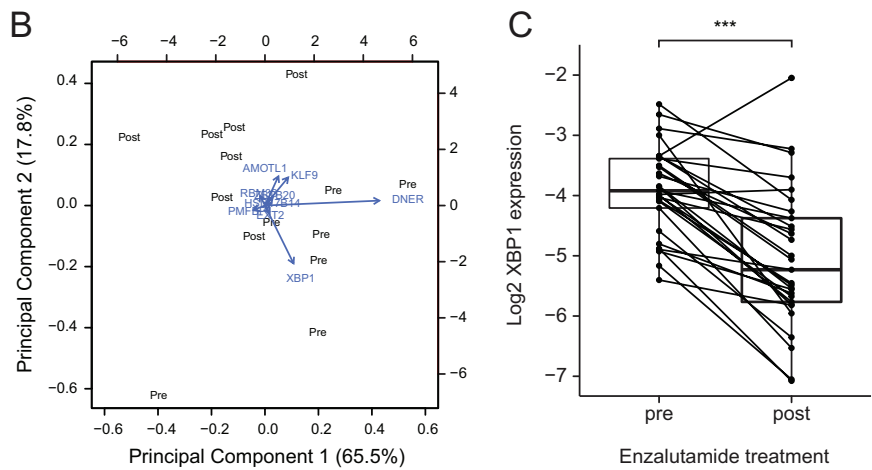

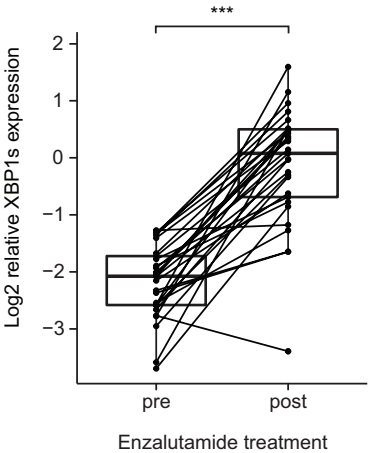

\section{Figure 1}

XBP1 expression is correlated with disease progression and response to hormone therapy. (A) Prognostic value of XBP1 expression represented by forest plot. The blue squares represent the hazard ratios of the individual studies and the horizontal lines the $95 \%$ confidence interval. The size of the squares is proportional to the number of patients in each study. The black diamond represents the overall hazard ratio. (B) Biplot of principal component analysis of the expression of the nine genes (AMOTL1, DNER, EXT2, HSD17B14, KLF9, PMFBP1, RBM33, XBP1 and ZBTB20) in tumors from patients before (pre) and after (post) androgen deprivation therapy (GSE48403). The arrows depict the contribution of each gene to the variance of the data. (C) Log2 XBP1 and relative XBP1s expression in samples from pre- and post-enzalutamide-treated patients $(n=29$, DARANA - NCT03297385). The points indicate the samples and the lines connect the paired pre- and post-therapy samples. ${ }^{*} \star * P$ value $<0.001$; paired $t$-test.

significantly increased upon 3 months of enzalutamide treatment (Fig. 1C). A similar trend was observed in the ADT-treated patients as well (Supplementary Fig. 1B), but it did not reach statistical significance possibly due to the small sample size $(n=7)$.

Cumulatively, we found that XBP1 is decreased after hormonal therapy and low levels are associated with biochemical progression, while no prognostic value of XBP1s was observed.

\section{XBP1 splicing is mediated by androgen receptor signaling}

Analyzing XBP1 transcript levels in clinical samples illustrated that XBP1 was altered upon androgen-targeted therapy. We therefore wondered whether XBP1 is a direct androgen-responsive gene. To assess this, hormonedeprived LNCaP cells were exposed to synthetic androgen R1881 for 8, 16 or $24 \mathrm{~h}$, after which RNA was isolated (Fig. 2A). Total XBP1 mRNA levels were slightly increased in a time-dependent manner, whereas the XBP1 spliced mRNA was significantly increased (Fig. 2A). As splicing of $\mathrm{XBP} 1$ is known to be mediated by the endoribonuclease ERN1 (Yoshida et al. 2001, Calfon et al. 2002), we also examined ERN1 expression. Upon exposure to R1881, transcript levels of ERN1 were elevated over time (Fig. 2A), concordant with a previous study (Sheng et al. 2015).

Expression and splicing of XBP1 was further confirmed in RNA-seq samples from LNCaP cells stimulated for $24 \mathrm{~h}$ with R1881 or $3 \mathrm{~h}$ with the ER stress inducer thapsigargin (Tg). The read count in exons and the splice junction reads were determined and plotted in Fig. 2B. Expression of XBP1 was increased upon R1881 or Tg exposure, and splicing was clearly strongly induced (Fig. 2B). In addition, R1881-induced expression and splicing of XBP1 was validated in VCaP cells (Supplementary Fig. 2A).

To assess the role of AR in the transcription regulation of both ERN1 and XBP1 as well as the subsequent splicing of XBP1, we knocked down AR expression. Quantification analyses showed that AR expression levels were reduced by 30-40\% (Fig. 2C). Knockdown of AR abrogates androgeninduced ERN1 and XBP1s expression. In contrast, the levels of total XBP1 mRNA were only slightly affected upon AR knockdown followed by short-term R1881 stimulation. Consistent with the mRNA data, Western blot analysis showed that AR knockdown prevented R1881-mediated increase of ERN1 and XBP1s protein levels (Fig. 2D and Supplementary Fig. 2C), while XBP1u protein levels remain relatively unaffected (Supplementary Fig. 2C). In addition, we observed AR-binding events in the first intron of ERN1 and in the region upstream of the XBP1 gene (Supplementary Fig. 2B). In conclusion, these results indicate that AR drives the expression of XBP1 and ERN1 and consequent splicing of XBP1.

\section{R1881- and Tg-induced XBP1s chromatin binding at genes involved in the unfolded protein response}

To identify the biological role of XBP1s in prostate cancer cells, we performed ChIP-seq for XBP1s in hormonedeprived LNCaP cells treated with either vehicle or R1881 for $24 \mathrm{~h}$. XBP1s ChIP-seq was also carried out under ER stress conditions ( $3 \mathrm{~h}$ of Tg stimulation), boosting XBP1s protein levels (Supplementary Fig. 3A). Two independent 

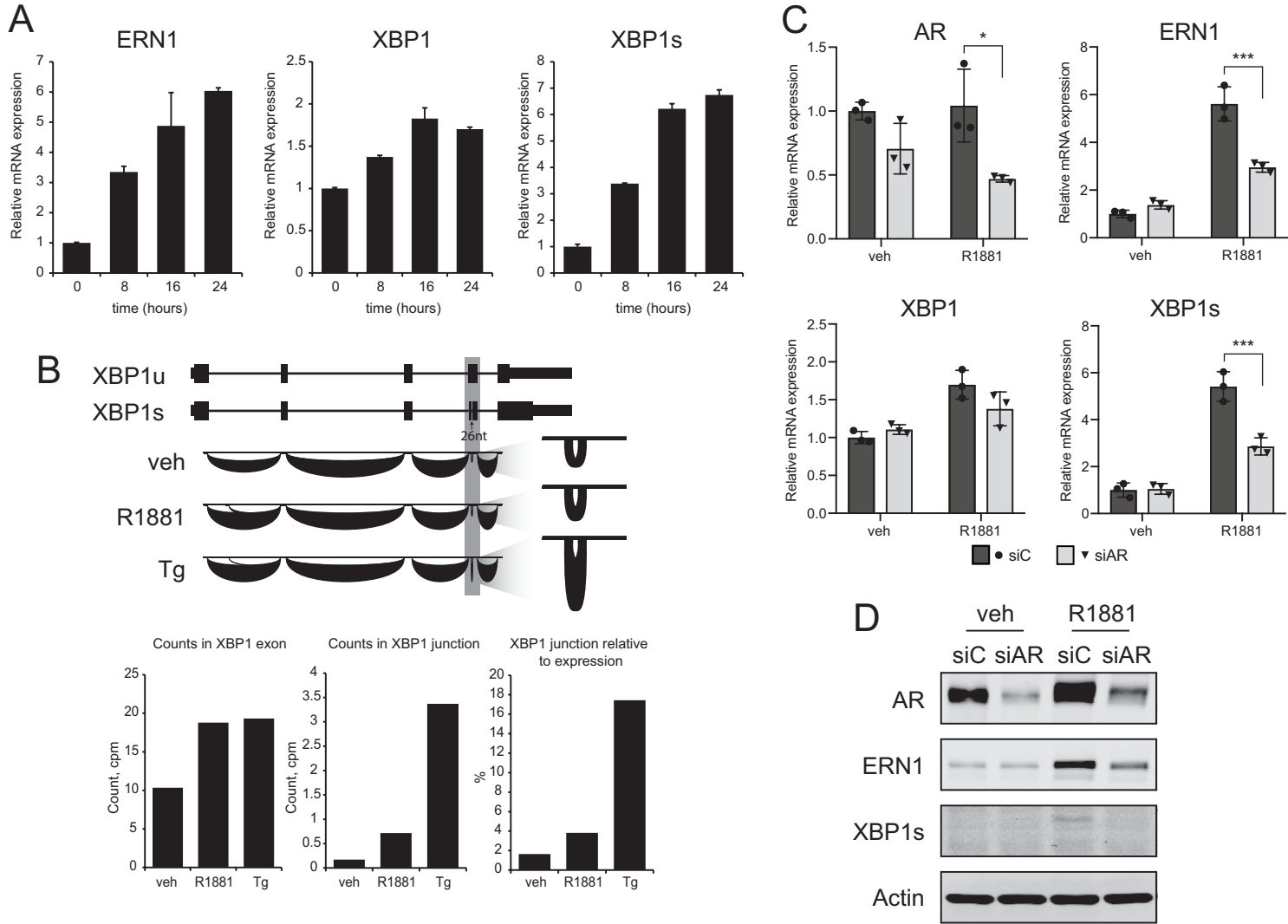

Figure 2

AR signaling triggers splicing of XBP1. (A) Relative mRNA expression levels of ERN1, XBP1 and XBP1s at 0, 8, 16 and $24 \mathrm{~h}$ after R1881 stimulation. Shown is a representative experiment of two independent biological replicates. Error bars indicate s.D. (B) Splice junction tracks. Top: Exon-intron structure of XBP1 $\mathrm{u}$ and XBP1s is shown. The arcs indicate splice junction reads, and the height of the arc is proportional to the read counts in that area. The splicing region of the 26 nucleotides (shaded area) is enlarged. Bottom: Quantification of XBP1 splicing in RNA-seq samples from LNCaP cells treated with vehicle, R1881 (24 h) or Thapsigargin (Tg, 3 h). Y-axis indicates counts per million (cpm), average of two biological replicates. (C) Relative mRNA expression levels of AR, ERN1, XBP1 and XBP1s. LNCaP cells were transfected with siControl (siC) or siAR and treated for $24 \mathrm{~h}$ with either vehicle (veh) or R1881. Shown are individual datapoints and mean of three independent biological replicates. Error bars indicate S.D. ${ }^{*} P$ value $<0.05$, $* * * P$ value $<0.001$; two-way ANOVA followed by Tukey's multiple comparisons test. (D) Western blot analysis for AR, ERN1 and XBP1s expression in hormone-deprived LNCaP cells transfected with siControl (siC) or siAR. Cells were vehicle- (veh) or R1881-treated for $24 \mathrm{~h}$. Actin was used as a loading control. A representative of three biological replicates is shown.

XBP1s ChIP-seq replicates were generated, which were highly correlated (Supplementary Fig. 3B and C). To rule out aspecific ChIP-seq signal coming from the XBP1s antibody, ERN1-knockout LNCaP cells were used as a negative control, as these cells do express XBP1 but are unable to splice the protein (Supplementary Fig. 4A and B). No XBP1s ChIP-seq signal was observed in ERN1knockout LNCaP cells (Supplementary Fig. 4C and D), confirming the specificity of the used XBP1s antibody in the ChIP experiments.

As exemplified at five genomic loci, XBP1s chromatin binding was induced upon R1881 and $\mathrm{Tg}$ exposure (Fig. 3A). Overexpression of XBP1s resulted in induced expression of these XBP1s-bound genes (Supplementary Fig. 5A). Besides induced peak intensity, the number of XBP1s peaks also increased from 702 in vehicle-treated cells to 3225 and 15030 peaks in R1881 and Tg conditions, respectively (Fig. 3B). The induced binding of XBP1s upon R1881 and Tg stimulation is likely to reflect the abundance of XBP1s protein (Supplementary Figs 3A and $4 \mathrm{~B})$. Next, we divided the XBP1s-binding sites in those present in all conditions (shared) and those that are either R1881-induced or Tg- gained. The shared sites displayed the strongest XBP1s binding as compared to R1881- and Tg-induced peaks (Fig. 3C, D and Supplementary Fig. 5B). In addition, the shared XBP1s peaks show enrichment at promoters, marked with strong H3K4me3 and H3K27ac signal, and absence of AR binding (Fig. 3C, E and F). Conversely, R1881-induced sites are enriched at intronic and distal intergenic regions, with strong signal of AR binding (Fig. 3C, E and F). In line with these results, nuclear hormone receptor family motifs are enriched in 

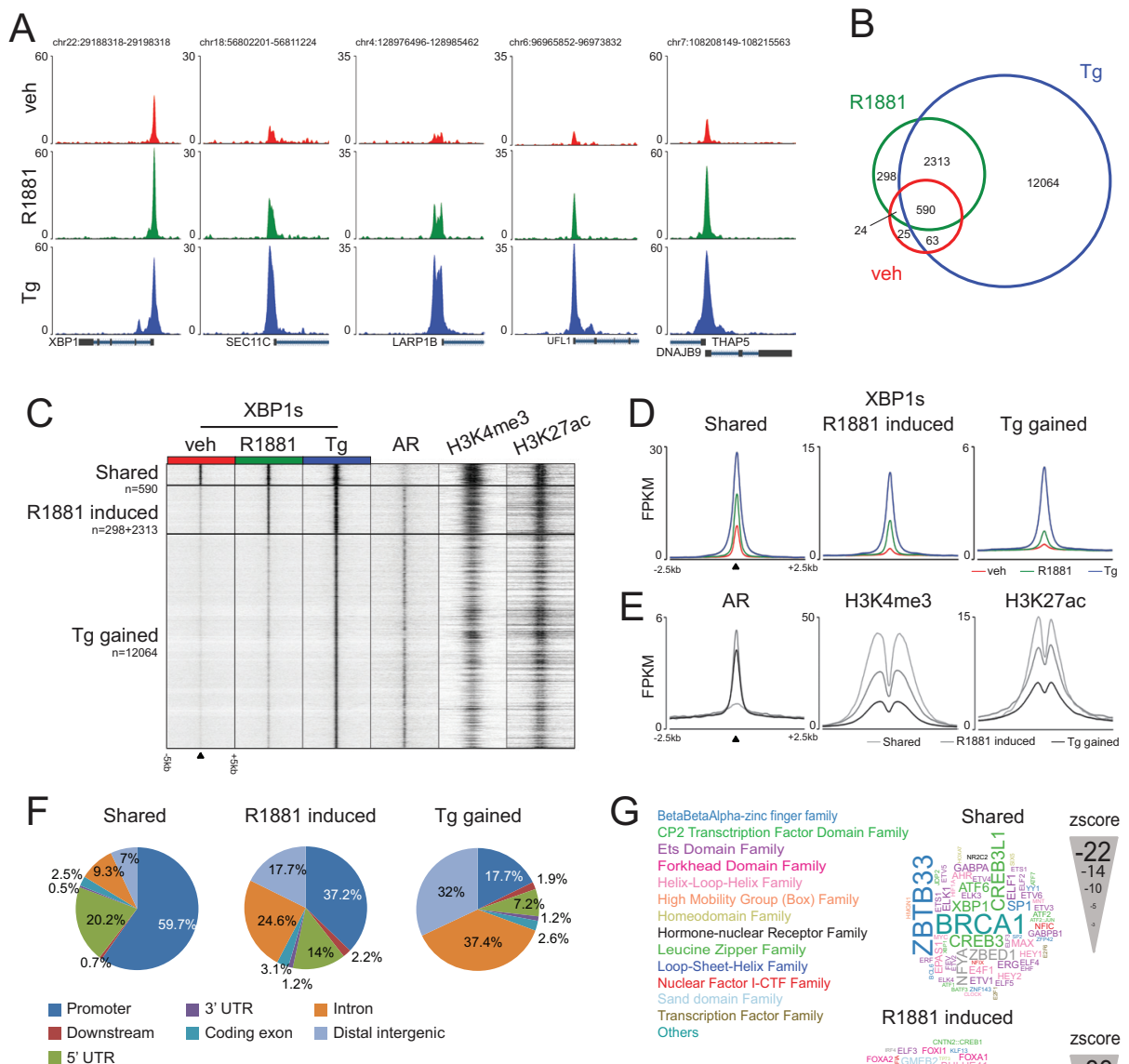

5' UTR

H Top Canonical pathways

Top Canor

Unfolded protein response

Unfolded protein

Endoplasmic Reticulum Stress Pathway Hypoxia Signaling in the Cardiovascular
Aldosterone Signaling in Epithelial Cells

Aldosterone Sig

EIF2 Signaling

Protein Ubiquitination Pathway

mTOR signaling

Regulation of elF4 and p70S6K Signaling

Proline Biosynthesis I

Tg gained

mTOR signaling

PISK/AKT Signaling

AMPK Signaling

$8.01 \mathrm{E}-06$

Pompounds $\quad 2.52 \mathrm{E}-05$

\begin{tabular}{lll} 
& \multicolumn{2}{l}{ Top Upstream Regulators } \\
& Shared & \\
$1.19 \mathrm{E}-13$ & ATF6 & $2.00 \mathrm{E}-08$ \\
$1.08 \mathrm{E}-10$ & RAB1B & $2.50 \mathrm{E}-08$ \\
$8.40 \mathrm{E}-07$ & XBP1 & $7.43 \mathrm{E}-07$ \\
$1.33 \mathrm{E}-04$ & ATP2A1 & $2.61 \mathrm{E}-06$ \\
$1.39 \mathrm{E}-04$ & OSBPL8 & $1.26 \mathrm{E}-05$ \\
& & \\
& R1881 induced & \\
$3.13 \mathrm{E}-09$ & MYC & $2.85 \mathrm{E}-05$ \\
$8.52 \mathrm{E}-07$ & E2F1 & $1.85 \mathrm{E}-04$ \\
$9.14 \mathrm{E}-06$ & KDM5B & $3.70 \mathrm{E}-04$ \\
$7.44 \mathrm{E}-05$ & 26s Proteasome & $5.25 \mathrm{E}-04$ \\
$3.99 \mathrm{E}-04$ & CST5 & $6.28 \mathrm{E}-04$ \\
& & \\
& Tg gained & \\
$1.90 \mathrm{E}-06$ & CST5 & $3.12 \mathrm{E}-10$ \\
$2.33 \mathrm{E}-06$ & TP53 & $7.35 \mathrm{E}-09$ \\
$8.01 \mathrm{E}-06$ & NUPR1 & $1.57 \mathrm{E}-08$ \\
$1.52 \mathrm{E}-05$ & MYC & $1.12 \mathrm{E}-07$ \\
$2.10 \mathrm{E}-05$ & TRAP1 & $1.15 \mathrm{E}-06$ \\
& &
\end{tabular}

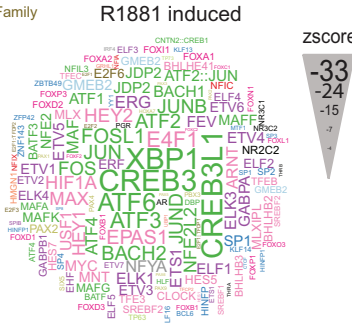

Tg gained

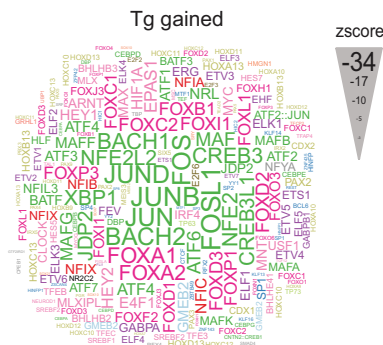

\section{Figure 3}

XBP1s chromatin binding in LNCaP. (A) Snapshots of XBP1s chromatin binding at five example loci for vehicle (red), R1881 (24 h, green) and Tg (3 h, blue) conditions. Genomic coordinates are indicated. (B) Venn diagram showing overlapping XBP1s-binding sites at indicated treatments (vehicle, red; R1881, green; Tg, blue). (C) Heatmap visualizing ChIP-seq signal (fragments per kilobase pair per million reads (FPKM)) in vehicle, R1881 and Tg conditions for XBP1s as well as AR, H3K4me3 and H3K27ac ChIP-seq signal from publicly available datasets. Data are centered at XBP1s peaks, depicting a 5-kb window around the peak. Subclassification of the binding events represent XBP1s sites shared under all conditions (shared, 590 binding sites), R1881-induced (298 + 2313 binding sites) and Tg-gained (12064 binding sites). (D) Average XBP1s signal (FPKM) at either shared, R1881-induced or Tg-gained regions. Data are centered at XBP1s peaks, depicting a 2.5-kb window around the peak. Vehicle-stimulated samples are shown in red, R1881-stimulated samples in green and Tg-stimulated samples in blue. (E) Average signal (FPKM) for AR, H3K4me3 and H3K27ac at the three XBP1s-binding clusters defined in C (Shared, R1881-induced and Tg-gained). (F) Genomic distribution of XBP1s binding at shared, R1881-induced and Tg-gained regions across genomic features. (G) Enrichment of motifs in the shared, R1881-induced and Tg-gained XBP1s binding sites. The font size represents the Z-score and colors correspond to transcription factor families. $(\mathrm{H})$ Top canonical pathways and top upstream regulators based on Ingenuity Pathway analysis of genes with proximal XBP1s binding. 
this cluster (Fig. 3G). As expected for XBP1s ChIP-seq, we found significant enrichment of the XBP1 motif consisting of an ACGT sequence in all clusters (Fig. 3G). Using the ReMap annotation tool containing a collection of various published ChIP-seq data sets (Cheneby et al. 2018), we found a number of transcription factors whose binding sites overlapped with XBP1s, including other bZIP transcription factors (e.g. FOS, JUN, CREB3), AR and FOXA1 (Supplementary Fig. 6A and B).

To identify XBP1s target genes, we scanned for genes with proximal XBP1s binding in a window of $20-\mathrm{kb}$ upstream or within their gene body. Using ingenuity pathway analysis, we found that XBP1s target genes were enriched for genes involved in the unfolded protein response, eIF2 signaling and protein ubiquitination with no apparent differences between the subclusters (Fig. 3H).

Taken together, these analyses revealed that XBP1s binds the chromatin largely at promoters in hormonedeprived conditions, while AR stimulation induces XBP1s binding at enhancers that are co-occupied by AR.

\section{Downregulation of androgen response genes and UPR genes upon knockdown of XBP1}

By exploring gene expression of vehicle- and R1881treated LNCaP cells, we identified 934 differentially expressed genes upon 24 h R1881 stimulation $(P$ value
$<0.05$ and absolute $\log _{2}$ fold change $>2.0$ ), including the ERN1 gene among others. Gene set enrichment analysis demonstrated positive enrichment for genes involved in the unfolded protein response (UPR) as well as (as expected) AR target genes (Fig. 4A and Supplementary Fig. $8 \mathrm{~A})$. While XBP1s overexpression leads to upregulation of XBP1s target genes, no such effect can be observed when overexpressing the unspliced protein (Supplementary Figs $5 \mathrm{~A}$ and $7 \mathrm{~A}, \mathrm{~B})$. AR target genes, however, remain unaffected when overexpressing either of the XBP1 variants (Supplementary Fig. 7A and B). Conversely, knockdown of XBP1 resulted in significant downregulation of AR target genes and unfolded protein response genes (Fig. 4B and Supplementary Fig. 8B, C). These results were successfully validated in an independent RNA-seq dataset of LNCaP cells with siRNA-mediated XBP1 silencing (Fig. 4C) (Sheng et al. 2019), and a similar trend could be observed in LNCaP cells treated with an RNase-specific ERN1 inhibitor (Supplementary Fig. 8D). In addition, upstream regulator analysis of the differentially expressed genes upon XBP1 knockdown revealed AR, dihydrotestosterone, ERN1 and $\mathrm{XBP} 1$ to be involved in transcriptional regulation of these genes (Fig. 4D). In conclusion, AR induces the expression of many genes including ERN1 and XBP1, enhancing XBP1s activity (Fig. 5). Subsequently, these events drive the expression of a subset of canonical AR-responsive genes along with activation of the unfolded protein response in prostate cancer cells (Fig. 5).
A
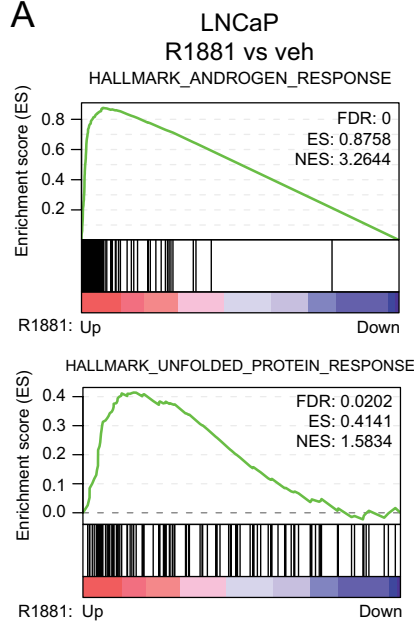

B
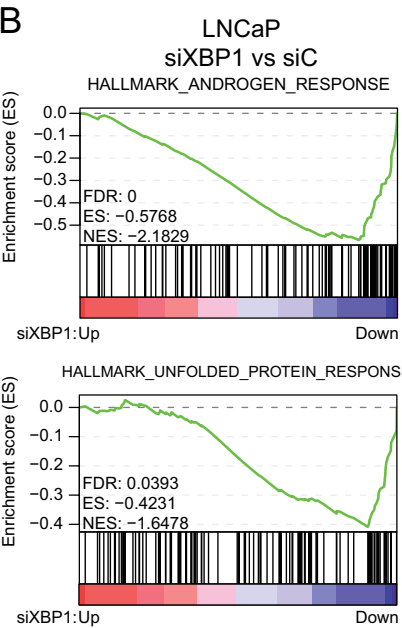

C
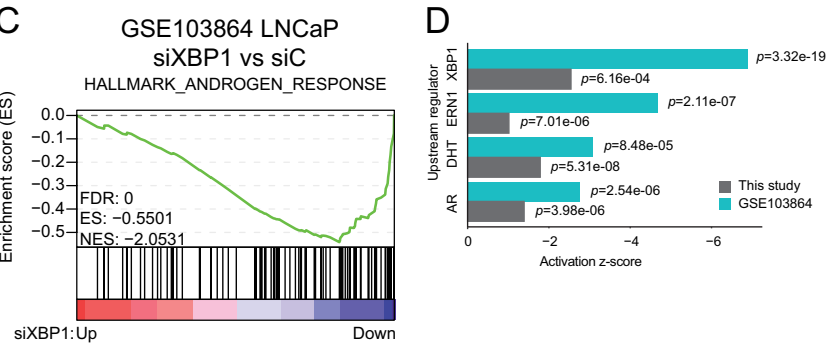

siXBP1:Up

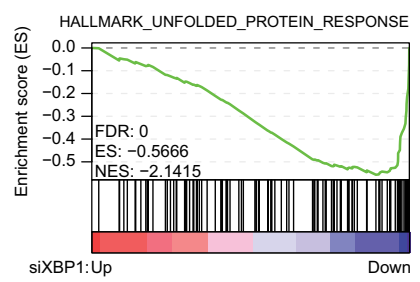

\section{Figure 4}

AR-XBP1 transcription regulation of androgen response and unfolded protein response genes. (A) GSEA results for the gene sets HALLMARK_ANDROGEN RESPONSE and HALLMARK_UNFOLDED_PROTEIN_RESPONSE using RNA-seq data from LNCaP cells treated with vehicle or R1881 for 24 h. NES, normalized enrichment score. (B) GSEA results for the gene sets HALLMARK_ANDROGEN_RESPONSE and HALLMARK_UNFOLDED_PROTEIN_RESPONSE using RNA-Seq data from LNCaP cells transfected with siControl (siC) or siXBP1. NES, normalized enrichment score. (C) GSEA results for the gene sets HALLMARK_ ANDROGEN_RESPONSE and HALLMARK_UNFOLDED_PROTEIN_RESPONSE using publicly available RNA-seq data from LNCaP cells transfected with siControl (siC) or siXBP1 (GSE103864). NES, normalized enrichment score. (D) Upstream regulators predicted by IPA analysis of differentially expressed genes upon XBP1 knockdown. The regulation z-score and the overlap $P$ value are shown for AR, dihydrotestosterone, ERN1 and XBP1. 


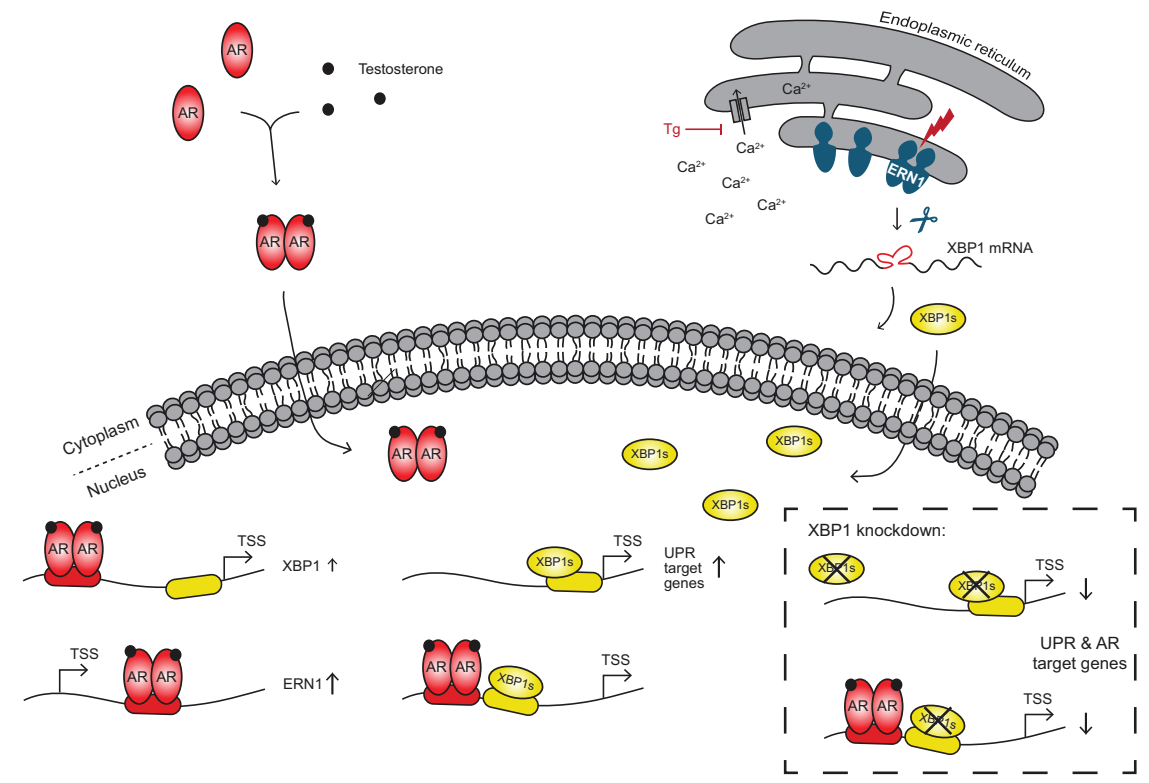

\begin{abstract}
Figure 5
Androgen-induced modulation of AR and XBP1s transcriptional activity. Testosterone binds to the androgen receptor (AR). Upon binding, AR dimerizes and translocates to the nucleus where it binds the chromatin, mainly at androgen response elements (AREs, red). AR binds within the intron of the ERN1 gene and enhancer proximal to $X B P 1$, ultimately leading to increased expression of ERN1 and XBP1. The AR-induced expression of ERN1 induces splicing of XBP1 (XBP1s). This subsequently leads to transcription of genes involved in, among others, the unfolded protein response (UPR). Conversely, knockdown of XBP1 results in downregulation of UPR, but also androgen-responsive genes, showing that XBP1 is functionally supporting part of the AR transcriptional program.
\end{abstract}

\section{Discussion}

Accumulating evidence has demonstrated that ER stress plays a crucial role in tumor growth and survival (Yadav et al. 2014). XBP1, a key transcription factor of the ER stress response, is often overexpressed in cancer and correlates with clinical outcome, as was previously reported for oral squamous cell carcinoma and hepatocellular carcinoma (Sun et al. 2018, Wu et al. 2018). Here, we report that in five independent cohorts, high XBP1 expression associates with better biochemical recurrence outcome in patients with prostate cancer. Even though these data suggest a connection between ER stress and clinical outcome, the spliced form is the accurate biomarker for activated ER stress. Furthermore, since the unspliced form negatively regulates XBP1s (Yoshida et al. 2006), biological interpretation of the clinical data is challenging. As the spliced mRNA showed no association with biochemical recurrence in the RNA-seq cohort (TCGA), these data suggest that XBP1 isoforms are differently associated with recurrence. Future studies should be aimed to further elucidate the clinical implications of both XBP1 isoforms. Further, as biochemical recurrence is a poor indicator of survival outcome, the potential impact of both XBP1 variants on disease-specific survival as a stronger clinical endpoint should be tested.

Previously, we reported that XBP1 mRNA levels are markedly increased in primary prostate cancer as compared to benign tissues and metastasis (Stelloo et al. 2015). The variable expression level of XBP1 at the different stages of the disease as well as decreased XBP1 upon hormone therapy (Fig. 1C) (Sowalsky et al. 2018) may be AR signaling dependent, as XBP1 is transcriptionally regulated by AR (Fig. 2) (Sheng et al. 2015). In concordance, Sowalsky and colleagues showed a positive correlation between AR activity score and XBP1 expression (Sowalsky et al. 2018). Also, in our cohort of prostate cancer patients receiving neoadjuvant enzalutamide therapy, as well as in an additional publicly available cohort, receiving ADT (Rajan et al. 2014), XBP1 expression levels were decreased, further confirming the transcriptional dependency of XBP1 on AR action. As XBP1s levels were increased after neoadjuvant enzalutamide, cells may experience stress following AR inhibition with a consequent induction of the UPR pathway. Further research should be aimed to investigate the physiological consequences of XBP1s induction after enzalutamide treatment, along with the potential clinical implications thereof.

Here we show, in agreement with previously published data, that both ERN1 as well as XBP1 are under direct transcriptional control of AR (Sheng et al. 2015), and that ERN1 is critically involved in the splicing of XBP1, yielding increased XBP1s levels (Fig. 2). Interestingly, even though we observed AR binding proximal to the $X B P 1$ locus in primary prostate cancers (Stelloo et al. 2015), XBP1s protein expression is primarily regulated at the level of splicing through AR-induced ERN1. As AR activation led to increased XBP1 splicing - a biomarker for UPR signaling - future research should address whether misfolded proteins do indeed accumulate in AR-stimulated prostate cancer cells.

Besides increased expression of AR target genes upon androgen stimulation, androgens also regulate the (c) 2020 Society for Endocrinology Published by Bioscientifica Ltd. Printed in Great Britain 
expression of ER stress-associated genes (Fig. 4) (Murray et al. 2004, Sheng et al. 2015). Most likely, XBP1s controls the expression of these genes, as XBP1s primarily binds at promoters of UPR genes leading to increased expression of these genes as shown by overexpression of XBP1s. In the absence of hormones, XBP1s binds the chromatin largely at promoters. In contrast, upon UPR induction through physiological (R1881) or supraphysiological (Tg) cues, the majority of XBP1s is bound to distal regulatory elements. This phenomenon is also observed upon liver damage (stress), where XBP1s occupies mostly promoters in shamoperated mice livers, while partial hepatectomy resulted in XBP1s binding mainly at distal elements (Argemi et al. 2017). At XBP1s-binding sites, DNA-binding motifs of the bZIP transcription factor family were enriched as well as many other transcription factor family motifs, suggesting that XBP1 cooperates with other transcription factors. In accordance with this, we found genomic overlap between AR- and XBP1-binding sites, and permuting these signaling cascades in prostate cancer cells illustrated the enrichment of the same gene sets involved in AR signaling and UPR pathways (Fig. 5). AR stimulation resulted in the upregulation of androgen- as well as UPR-response pathways, while XBP1 knockdown showed, in effect, the exact opposite phenotype. As XBP1 acts downstream of $\mathrm{AR}$, the XBP1 signaling cascade may provide a possible feedback mechanism to refine the activity for part of the canonical AR-responsive gene repertoire. However, XBP1 overexpression alone did not lead to upregulation of AR-target genes, suggesting that XBP1's supportive role at these sites remains dependent on AR activation. Previously published endogenous proteomic profiling of AR in prostate cancer models suggests no direct proteinprotein interaction between AR and XBP1, since no XBP1 peptides were detected (Stelloo et al. 2018). Even though this mass spectrometry technology is quite sensitive, negative data are hard to interpret and a possible interaction can formally not be excluded. In addition, while most of our results point toward a functional role of XBP1s in AR biology, we cannot rule out potential involvement of the unspliced XBP1 isoform at this stage. Future studies should be undertaken to fully elucidate the relative contribution of each XBP1 variant in this process.

Taken together, we present a multi-step mechanism of gene regulation, in which androgen-induced expression of ERN1 enhances XBP1 splicing and transcriptional activity. This signaling cascade may prepare the cells for the increased translation, protein (mis)folding and mRNA decay that accompanies AR-regulated tumor cell proliferation.

\section{Supplementary materials}

This is linked to the online version of the paper at https://doi.org/10.1530/ ERC-19-0181.

\section{Declaration of interest}

W $\mathrm{Z}, \mathrm{A} M \mathrm{M}$ and $\mathrm{H} \mathrm{v}$ d $\mathrm{P}$ received research funding from Astellas Pharma. The other authors declare that there is no conflict of interest that could be perceived as prejudicing the impartiality of this article.

\section{Funding}

This work was supported by Movember (NKI01 to A M B and W Z); KWF Dutch Cancer Society (10084 ALPE to A M B and W Z); KWF Dutch Cancer Society/Alpe d'HuZes Bas Mulder Award (NKI 2014-6711 to W Z); Netherlands Organization for Scientific Research (NWO-VIDI-016.156.401 to $W$ Z).

\section{Acknowledgements}

The authors would like to acknowledge the NKI-AVL Core Facility Molecular Pathology \& Biobanking (CFMPB) for supplying NKI-AVL Biobank material and/or lab support, the NKI Genomics Core Facility for Illumina sequencing and bioinformatics support and the NKI Research High Performance Computing (RHPC) facility for computational infrastructure. They also thank Zwart/Bergman lab members for helpful discussions and technical advice.

\section{References}

Acosta-Alvear D, Zhou Y, Blais A, Tsikitis M, Lents NH, Arias C, Lennon CJ, Kluger Y \& Dynlacht BD 2007 XBP1 controls diverse cell type- and condition-specific transcriptional regulatory networks. Molecular Cell 27 53-66. (https://doi.org/10.1016/j. molcel.2007.06.011)

Argemi J, Kress TR, Chang HCY, Ferrero R, Bertolo C, Moreno H, Gonzalez-Aparicio M, Uriarte I, Guembe L, Segura V, et al. 2017 $\mathrm{X}$-box binding protein 1 regulates unfolded protein, acute-phase, and DNA damage responses during regeneration of mouse liver. Gastroenterology 152 1203.e15-1216.e15. (https://doi.org/10.1053/j. gastro.2016.12.040)

Boormans JL, Korsten H, Ziel-van der Made AJ, van Leenders GJ, de Vos CV, Jenster G \& Trapman J 2013 Identification of TDRD1 as a direct target gene of ERG in primary prostate cancer. International Journal of Cancer 133 335-345. (https://doi.org/10.1002/ijc.28025)

Brinkman EK, Chen T, Amendola M \& van Steensel B 2014 Easy quantitative assessment of genome editing by sequence trace decomposition. Nucleic Acids Research 42 e168. (https://doi. org/10.1093/nar/gku936)

Calfon M, Zeng H, Urano F, Till JH, Hubbard SR, Harding HP, Clark SG \& Ron D 2002 IRE1 couples endoplasmic reticulum load to secretory capacity by processing the XBP-1 mRNA. Nature 415 92-96. (https:// doi.org/10.1038/415092a)

Cancer Genome Atlas Research Network 2015 The molecular taxonomy of primary prostate cancer. Cell 163 1011-1025. (https://doi. org/10.1016/j.cell.2015.10.025)

Chen X, Iliopoulos D, Zhang Q, Tang Q, Greenblatt MB, Hatziapostolou M, Lim E, Tam WL, Ni M, Chen Y, et al. 2014 XBP1 promotes triple-negative breast cancer by controlling the HIF1alpha pathway. Nature 508 103-107. (https://doi.org/10.1038/nature13119) 
Cheneby J, Gheorghe M, Artufel M, Mathelier A \& Ballester B 2018 ReMap 2018: an updated atlas of regulatory regions from an integrative analysis of DNA-binding ChIP-seq experiments. Nucleic Acids Research 46 D267-D275. (https://doi.org/10.1093/nar/gkx1092)

Cuperlovic-Culf M, Belacel N, Davey M \& Ouellette RJ 2010 Multi-gene biomarker panel for reference free prostate cancer diagnosis: determination and independent validation. Biomarkers 15 693-706. (https://doi.org/10.3109/1354750X.2010.511268)

Duarte M, Vende P, Charpilienne A, Gratia M, Laroche C \& Poncet D 2019 Rotavirus infection alters splicing of the stress-related transcription factor XBP1. Journal of Virology 93 e01739-18. (https:// doi.org/10.1128/JVI.01739-18)

Erzurumlu Y \& Ballar P 2017 Androgen mediated regulation of endoplasmic reticulum-associated degradation and its effects on prostate cancer. Scientific Reports 7 40719. (https://doi.org/10.1038/ srep40719)

Glinsky GV, Glinskii AB, Stephenson AJ, Hoffman RM \& Gerald WL 2004 Gene expression profiling predicts clinical outcome of prostate cancer. Journal of Clinical Investigation 113 913-923. (https://doi. org/10.1172/JCI20032)

Gulzar ZG, McKenney JK \& Brooks JD 2013 Increased expression of NuSAP in recurrent prostate cancer is mediated by E2F1. Oncogene 32 70-77. (https://doi.org/10.1038/onc.2012.27)

Harmsen T, Klaasen S, van de Vrugt H \& Te Riele H 2018 DNA mismatch repair and oligonucleotide end-protection promote base-pair substitution distal from a CRISPR/Cas9-induced DNA break. Nucleic Acids Research 46 2945-2955. (https://doi.org/10.1093/nar/gky076)

Hassler JR, Scheuner DL, Wang S, Han J, Kodali VK, Li P, Nguyen J, George JS, Davis C, Wu SP, et al. 2015 The IRE1alpha/XBP1s pathway is essential for the glucose response and protection of beta cells. PLoS Biology 13 e1002277. (https://doi.org/10.1371/journal.pbio.1002277)

Hetz C, Chevet E \& Harding HP 2013 Targeting the unfolded protein response in disease. Nature Reviews: Drug Discovery 12 703-719. (https://doi.org/10.1038/nrd3976)

Huo JS, McEachin RC, Cui TX, Duggal NK, Hai T, States DJ \& Schwartz J 2006 Profiles of growth hormone $(\mathrm{GH})$-regulated genes reveal timedependent responses and identify a mechanism for regulation of activating transcription factor 3 by GH. Journal of Biological Chemistry 281 4132-4141. (https://doi.org/10.1074/jbc.M508492200)

Kumar V, Muratani M, Rayan NA, Kraus P, Lufkin T, Ng HH \& Prabhakar S 2013 Uniform, optimal signal processing of mapped deep-sequencing data. Nature Biotechnology 31 615-622. (https://doi. org/10.1038/nbt.2596)

Lee AH, Iwakoshi NN \& Glimcher LH 2003 XBP-1 regulates a subset of endoplasmic reticulum resident chaperone genes in the unfolded protein response. Molecular and Cellular Biology 23 7448-7459. (https://doi.org/10.1128/mcb.23.21.7448-7459.2003)

Lerdrup M, Johansen JV, Agrawal-Singh S \& Hansen K 2016 An interactive environment for agile analysis and visualization of ChIPsequencing data. Nature Structural and Molecular Biology 23 349-357. (https://doi.org/10.1038/nsmb.3180)

Murray JI, Whitfield ML, Trinklein ND, Myers RM, Brown PO \& Botstein D 2004 Diverse and specific gene expression responses to stresses in cultured human cells. Molecular Biology of the Cell $\mathbf{1 5}$ 2361-2374. (https://doi.org/10.1091/mbc.e03-11-0799)

Rajan P, Sudbery IM, Villasevil ME, Mui E, Fleming J, Davis M, Ahmad I, Edwards J, Sansom OJ, Sims D, et al. 2014 Next-generation sequencing of advanced prostate cancer treated with androgendeprivation therapy. European Urology 66 32-39. (https://doi. org/10.1016/j.eururo.2013.08.011)

Ritchie ME, Phipson B, Wu D, Hu Y, Law CW, Shi W \& Smyth GK 2015 Limma powers differential expression analyses for RNA-sequencing and microarray studies. Nucleic Acids Research 43 e47. (https://doi. org/10.1093/nar/gkv007)

Robinson MD, McCarthy DJ \& Smyth GK 2010 edgeR: a Bioconductor package for differential expression analysis of digital gene expression data. Bioinformatics 26 139-140. (https://doi.org/10.1093/ bioinformatics/btp616)

Ross-Innes CS, Stark R, Teschendorff AE, Holmes KA, Ali HR, Dunning MJ, Brown GD, Gojis O, Ellis IO, Green AR, et al. 2012 Differential oestrogen receptor binding is associated with clinical outcome in breast cancer. Nature 481 389-393. (https://doi. org/10.1038/nature10730)

Sengupta S, Sharma CG \& Jordan VC 2010 Estrogen regulation of X-box binding protein-1 and its role in estrogen induced growth of breast and endometrial cancer cells. Hormone Molecular Biology and Clinical Investigation 2 235-243. (https://doi.org/10.1515/HMBCI.2010.025)

Sheng X, Arnoldussen YJ, Storm M, Tesikova M, Nenseth HZ, Zhao S, Fazli L, Rennie P, Risberg B, Waehre H, et al. 2015 Divergent androgen regulation of unfolded protein response pathways drives prostate cancer. EMBO Molecular Medicine 7 788-801. (https://doi. org/10.15252/emmm.201404509)

Sheng X, Nenseth HZ, Qu S, Kuzu OF, Frahnow T, Simon L, Greene S, Zeng Q, Fazli L, Rennie PS, et al. 2019 IRE1alpha-XBP1s pathway promotes prostate cancer by activating c-MYC signaling. Nature Communications 10 323. (https://doi.org/10.1038/s41467-018-08152-3)

Shin H, Liu T, Manrai AK \& Liu XS 2009 CEAS: cis-regulatory element annotation system. Bioinformatics 25 2605-2606. (https://doi. org/10.1093/bioinformatics/btp479)

Sowalsky AG, Ye H, Bhasin M, Van Allen EM, Loda M, Lis RT, MontaserKouhsari L, Calagua C, Ma F, Russo JW, et al. 2018 Neoadjuvantintensive androgen deprivation therapy selects for prostate tumor foci with diverse subclonal oncogenic alterations. Cancer Research $\mathbf{7 8}$ 4716-4730. (https://doi.org/10.1158/0008-5472.CAN-18-0610)

Stelloo S, Nevedomskaya E, van der Poel HG, de Jong J, van Leenders GJ, Jenster G, Wessels LF, Bergman AM \& Zwart W 2015 Androgen receptor profiling predicts prostate cancer outcome. EMBO Molecular Medicine 7 1450-1464. (https://doi.org/10.15252/emmm.201505424)

Stelloo S, Nevedomskaya E, Kim Y, Hoekman L, Bleijerveld OB, Mirza T, Wessels LFA, van Weerden WM, Altelaar AFM, Bergman AM, et al. 2018 Endogenous androgen receptor proteomic profiling reveals genomic subcomplex involved in prostate tumorigenesis. Oncogene 37 313-322. (https://doi.org/10.1038/onc.2017.330)

Sun Y, Jiang F, Pan Y, Chen X, Chen J, Wang Y, Zheng X \& Zhang J 2018 XBP1 promotes tumor invasion and is associated with poor prognosis in oral squamous cell carcinoma. Oncology Reports $\mathbf{4 0}$ 988-998. (https://doi.org/10.3892/or.2018.6498)

Takahashi S, Suzuki S, Inaguma S, Ikeda Y, Cho YM, Nishiyama N, Fujita T, Inoue T, Hioki T, Sugimura Y, et al. 2002 Down-regulation of human X-box binding protein 1 (hXBP-1) expression correlates with tumor progression in human prostate cancers. Prostate 50 154-161. (https://doi.org/10.1002/pros.10044)

Taylor BS, Schultz N, Hieronymus H, Gopalan A, Xiao Y, Carver BS, Arora VK, Kaushik P, Cerami E, Reva B, et al. 2010 Integrative genomic profiling of human prostate cancer. Cancer Cell 18 11-22. (https://doi.org/10.1016/j.ccr.2010.05.026)

Thorpe JA \& Schwarze SR 2010 IRE1alpha controls cyclin A1 expression and promotes cell proliferation through XBP-1. Cell Stress and Chaperones 15 497-508. (https://doi.org/10.1007/s12192-009-0163-4)

Wu S, Du R, Gao C, Kang J, Wen J \& Sun T 2018 The role of XBP1s in the metastasis and prognosis of hepatocellular carcinoma. Biochemical and Biophysical Research Communications 500 530-537. (https://doi.org/10.1016/j.bbrc.2018.04.033)

Yadav RK, Chae SW, Kim HR \& Chae HJ 2014 Endoplasmic reticulum stress and cancer. Journal of Cancer Prevention 19 75-88. (https://doi. org/10.15430/JCP.2014.19.2.75)

Yoshida H, Matsui T, Yamamoto A, Okada T \& Mori K 2001 XBP1 mRNA is induced by ATF6 and spliced by IRE1 in response to ER stress to produce a highly active transcription factor. Cell 107 881-891. (https://doi.org/10.1016/s0092-8674(01)00611-0)

Yoshida H, Oku M, Suzuki M \& Mori K 2006 pXBP1(U) encoded in XBP1 pre-mRNA negatively regulates unfolded protein response https://erc.bioscientifica.com

https://doi.org/10.1530/ERC-19-0181 (c) 2020 Society for Endocrinology Published by Bioscientifica Ltd. Printed in Great Britain 
activator pXBP1(S) in mammalian ER stress response. Journal of Cell Biology 172 565-575. (https://doi.org/10.1083/jcb.200508145)

Zambelli A, Mongiardini E, Villegas SN, Carri NG, Boot-Handford RP \& Wallis GA 2005 Transcription factor XBP-1 is expressed during osteoblast differentiation and is transcriptionally regulated by parathyroid hormone (PTH). Cell Biology
International 29 647-653. (https://doi.org/10.1016/j. cellbi.2005.03.018)

Zhang Y, Liu T, Meyer CA, Eeckhoute J, Johnson DS, Bernstein BE, Nusbaum C, Myers RM, Brown M, Li W, et al. 2008 Model-based analysis of ChIP-Seq (MACS). Genome Biology 9 R137. (https://doi. org/10.1186/gb-2008-9-9-r137)

Received in final form 26 November 2019

Accepted 5 December 2019

Accepted Manuscript published online 5 December 2019 (c) 2020 Society for Endocrinology Published by Bioscientifica Ltd. Printed in Great Britain 